\title{
SOLUÇÃO DO SOLO E ANÁLISE DE COMPONENTES PRINCIPAIS PARA MONITORAMENTO DA APLICAÇÃO DE LODO DE ESGOTO ${ }^{(1)}$
}

\author{
Aline Renée Coscione ${ }^{(2)}$, Livia Fernanda Mendonça Silva ${ }^{(3)}$, Isabella Clerici De Maria ${ }^{(2)}$, \\ Cristiano Alberto de Andrade ${ }^{(4)} \&$ Vera Lucia Ferracini ${ }^{(4)}$
}

\section{RESUMO}

O uso agrícola do lodo é uma alternativa viável para a reciclagem de nutrientes, embora haja certa preocupação com o aumento de espécies químicas solúveis originadas da mineralização da fração orgânica. Assim, para conhecer a disponibilidade de nutrientes e o risco de contaminação do solo e da água, o estudo da solução do solo é uma ferramenta valiosa. Nesses estudos, o número de variáveis utilizadas ultrapassa facilmente uma dezena, e ferramentas de análise exploratória, como a análise de componentes principais (ACP), revelam a existência ou não de amostras anômalas, de relações entre as variáveis medidas e sua contribuição relativa e de relações ou agrupamentos entre amostras. $\mathrm{O}$ objetivo deste trabalho foi identificar variáveis relacionadas com a solução do solo e alteradas, em razão do uso do lodo de esgoto e da adubação mineral, de forma a complementar os procedimentos para monitoramento/avaliação de áreas que recebem esse tipo de resíduo. A composição da solução do solo de uma área que recebeu lodo de esgoto por sete anos consecutivos foi estudada dos 22 aos 46 meses após a sua última aplicação. Com a solução do solo foi possível, por meio da ACP, distinguir as áreas em função da adubação aplicada. Após quatro anos da interrupção das aplicações sucessivas de lodo de esgoto, não verificou-se concentração apreciável de metais na solução do solo, embora ainda seja possível identificar traços da mineralização do resíduo. As variáveis $\mathrm{pH}, \mathrm{Mn}^{2+}, \mathrm{COD}, \mathrm{SO}_{4}{ }^{2-}, \mathrm{NO}_{3}{ }^{-}$e $\mathrm{NH}_{4}{ }^{+}$na solução do solo podem ser utilizadas no monitoramento de áreas tratadas com lodo de esgoto.

Termos de indexação: metais pesados, matéria orgânica, mineralização, análise multivariada.

\footnotetext{
(1) Parte da Dissertação de Mestrado da $2^{\text {a }}$ autora. Recebido para publicação em 19 de setembro de 2013 e aprovado em 12 de junho de 2014.

(2) Pesquisadora Cientifica, Centro de Solos e Recursos Ambientais, Instituto Agronômico - IAC. Av. Barão de Itapura, 1481. CEP 13020-902 Campinas (SP), Brasil. E-mail: aline@iac.sp.gov.br, icdmaria@iac.sp.gov.br

(3) Mestranda, Centro de Solos e Recursos Ambientais, IAC. E-mail: livia.fms@hotmail.com

(4) Pesquisador(a), Embrapa Meio Ambiente. Rodovia SP 340, km 127,5. Caixa Postal 69. CEP 13820-000 Jaguariúna (SP), Brasil. E-mail: cristiano.andrade@embrapa.br, vera.ferracini@embrapa.br
} 


\title{
SUMMARY: SOIL SOLUTION AND PRINCIPAL COMPONENT ANALYSIS FOR THE MONITORING OF AGRICULTURAL AREAS AMENDED WITH SEWAGE SLUDGE
}

\begin{abstract}
The use of sewage sludge in agriculture is a sound alternative for nutrient recycling, but the concern over increase in soluble chemical species from mineralization of organic matter remains. The study of the soil solution is a valuable tool for understanding the availability of nutrients and for assessing the risk of soil and water contamination. For such studies, where the number of parameters examined is usually more than 10, the use of multivariate analysis tools, such as principal component analysis (PCA), are quite useful in revealing outliers, the contribution of individuals and groups of parameters, and the relationship within tested samples. The objective of this study was to identify the parameters related to changes in the soil solution associated with the use of sewage sludge or mineral fertilization in order to complement procedures for monitoring/ assessing areas amended with them. The soil solution of an area which received sewage sludge for seven consecutive years was studied from 22 to 46 months after its last application. By using PCA it was possible to identify the treatments throughout the soil solution composition. Even four years after interrupting the successive applications of sewage sludge, the heavy metals in the soil solution of the area were close to the instrumental detection limits. However, changes in the soil solution composition due to mineralization of the remaining organic matter from the sewage sludge were still detectable. Based on those results, we propose a set of parameters for monitoring the soil solution of areas amended by sewage sludge: $\mathrm{pH}, \mathrm{Mn}^{+2}, \mathrm{COD}, \mathrm{SO}_{4}{ }^{2-}, \mathrm{NO}_{3}{ }^{-}$, and $\mathrm{NH}_{4}{ }^{+}$.
\end{abstract}

Index terms: heavy metals, organic matter, mineralization, multivariate analysis.

\section{INTRODUÇÃO}

O uso de resíduos orgânicos na agricultura, incluindo o lodo de esgoto (LE), tem se consagrado como prática ambientalmente correta em razão da possibilidade de reciclagem de nutrientes. Essa prática minimiza o impacto ambiental negativo do descarte desse material em aterros e economiza recursos naturais explorados para a fabricação de fertilizantes minerais. No entanto, na utilização desse resíduo como fonte de nutrientes para as culturas, deve-se considerar que a disponibilidade desses depende da mineralização da matéria orgânica. Já os fertilizantes minerais, geralmente solúveis em água, apresentam ao menos uma fração do nutriente prontamente disponível (Camargo \& Bettiol, 2010).

Em geral, lodos de esgoto contêm de 40 a $80 \%$ de matéria orgânica, $3 \%$ de N, $2 \%$ de P e $1 \%$ de K, além de micronutrientes como $\mathrm{Zn}, \mathrm{Cu}, \mathrm{Fe}, \mathrm{Mn}$ e Mo (Bettiol \& Camargo, 2006). Diversos trabalhos demonstraram os efeitos benéficos do lodo nas propriedades físicas, químicas e biológicas do solo (Simonete et al., 2003; De Maria et al., 2007; Bueno et al., 2011), com reflexos na nutrição das culturas e, consequentemente, na produtividade.

A Resolução n ${ }^{\circ} 375$ do Conama, publicada em 2006, define critérios e procedimentos para o uso agrícola de lodos de esgoto gerados em estações de tratamento de esgoto sanitários e seus produtos derivados, subsidiando a fiscalização pelo IBAMA e pelos órgãos ambientais e promovendo o uso adequado desse resíduo, a fim de prevenir a contaminação do solo e dos recursos hídricos e os possíveis efeitos não desejados à saúde da população (Conama, 2006). De acordo com esta resolução, lodos de esgoto para uso agrícola devem respeitar limites máximos de concentração de metais (teores totais) e patógenos; a aplicação do lodo é realizada considerando uma taxa fixa de mineralização do $\mathrm{N}$ total do resíduo em razão do processo de tratamento, supondo que nenhuma aplicação anterior tenha sido realizada na área que receberá o lodo. Também, devem-se monitorar periódica e obrigatoriamente componentes de qualidade do resíduo e das áreas de sua aplicação (análises de fertilidade e de teores totais de metais no solo). No momento em que foram criados os estudos, no longo prazo, em condições edafoclimáticas brasileiras, esses eram incipientes para servirem como única base para o estabelecimento de uma norma nacional. A fim de permitir o aprimoramento da regulamentação a partir do conhecimento científico e do desenvolvimento tecnológico atingidos, na própria resolução foi prevista sua revisão para sete anos após sua publicação, o que ainda não ocorreu (setembro de 2014).

Se por um lado o uso agrícola do lodo representa alternativa viável para reciclagem de nutrientes e menor pressão ambiental, na medida em que poupa recursos naturais, há também certa preocupação em razão da possibilidade de aumento de espécies químicas solúveis originadas do processo de mineralização da fração orgânica que podem atingir teores fitotóxicos, apresentar efeitos indesejáveis de acumulação nas plantas (Nogueira et al., 2010a) e causar poluição das águas subsuperficiais (Oliveira et al., 2001; Egiarte et al., 2005; Dynia et al., 2006). Assim, para o conhecimento da disponibilidade de nutrientes e do potencial risco de contaminação do solo e da água, o estudo da solução do solo é uma ferramenta valiosa. Estudos nesse sentido ainda são escassos e, na maioria das vezes, fazem-se inferências ou estimativas com 
base nos teores totais das espécies químicas e, ou, nos teores extraídos com auxílio de extratores químicos. Ainda mais raros são os estudos desenvolvidos em campo sob condições edafoclimáticas tropicais e em áreas com uso agrícola de lodo por diversos anos (Merlino et al., 2010; Nogueira et al., 2010b). O efeito residual desse lodo nas espécies químicas presentes na solução do solo também é fator importante a ser considerado e muito tem a contribuir para o conhecimento cientifico, a partir do qual são estabelecidos os critérios regulatórios para o uso agrícola desse tipo de resíduo (Conama, 2006).

Para estudos da solução do solo, em que o número de variáveis para avaliação ultrapassa facilmente uma dezena, ferramentas de análise exploratória como a análise de componentes principais (ACP) permitem revelar a existência ou não de amostras anômalas, de relações entre as variáveis medidas e de relações ou agrupamentos entre amostras (Lyra et al., 2010). A ACP pode ser utilizada também como ferramenta de compressão, uma vez que reduz a dimensionalidade da matriz de dados originais, pois as primeiras componentes principais têm maior contribuição das variáveis que apresentam maior variância no conjunto das medidas experimentais realizadas, o que consequentemente facilita a visualização dos dados. Dessa forma, a ACP pode ser utilizada para a identificação de um conjunto mínimo de variáveis ou medições necessárias para explicar a variância de determinado conjunto de dados. Torna-se necessário, entretanto, lembrar que as variáveis excluídas nem sempre apresentam resultados baixos, mas apenas não contribuem para mostrar as diferenças entre os tratamentos na área estudada.

Com exemplo de uso da ACP em área de pesquisa correlata a deste trabalho, pode-se citar Hadlich \& Ucha (2010). Esses autores avaliaram a distribuição de Cd na fase sólida de um solo cultivado na Bretanha, França, assim como os fatores que influenciaram sua disponibilidade. Para isso, foi avaliada a concentração total de $\mathrm{Cd}$ em 22 amostras, as quais variaram de 0,13 a $0,37 \mathrm{mg} \mathrm{kg}^{-1}$. Pela ACP, percebeu-se o efeito sinérgico do $\mathrm{P}_{2} \mathrm{O}_{5}$ na retenção de $\mathrm{Cd}$ nos solos cultivados. Considerando que o manejo agrícola com agroquímicos pode contaminar o solo com metais pesados, Valladares et al. (2009) utilizaram a ACP e técnicas de geoprocessamento para identificar a origem dos metais pesados $\mathrm{Cu}, \mathrm{Fe}, \mathrm{Mn}, \mathrm{Zn}, \mathrm{Ni}, \mathrm{Pb}, \mathrm{Cr}$ e $\mathrm{Cd}$ como contaminantes potenciais em solos agrícolas, em área cultivada com vinhedos no Estado de São Paulo. A ACP indicou enriquecimento do solo com $\mathrm{Cu}$ e $\mathrm{Zn}$ em razão do uso e manejo dos vinhedos com agroquímicos em décadas anteriores.

Neste trabalho, estudou-se por meio de ACP a composição da solução do solo dos 22 até os 46 meses após a última aplicação de lodo de esgoto ou fertilizante mineral, utilizado anualmente por sete anos consecutivos. O objetivo deste trabalho foi identificar variáveis relacionadas com a solução do solo e alteradas em razão do uso do lodo de esgoto, de forma a complementar procedimentos para monitoramento/ avaliação de áreas que recebem esse tipo de resíduo.

\section{MATERIAL E MÉTODOS}

\section{Área experimental}

A área experimental está localizada no Centro Experimental Central do Instituto Agronômico (IAC), na Fazenda Santa Eliza, no município de Campinas, SP. O solo é classificado como Latossolo Vermelho eutroférrico, de textura argilosa (Embrapa, 2006). Essa área recebeu aplicações de lodo de esgoto (LE) ou fertilizante mineral nitrogenado anualmente no período de 2001 a 2007, com três diferentes tratamentos (AM, 1L e 2L), em que AMé o tratamento sem aplicação de lodo, mas com fertilização com $\mathrm{N}$ mineral, sendo sulfato de amônio no plantio e ureia em cobertura $\left(\mathrm{AM}=80 \mathrm{~kg} \mathrm{ha}^{-1} \mathrm{ano}^{-1}\right.$ de $\left.\mathrm{N}\right) ; 1 \mathrm{~L}$ é a aplicação de uma vez a dose recomendada de $\mathrm{N}$ via lodo $\left(1 \mathrm{~L} \approx 10 \mathrm{t} \mathrm{ha}^{-1} \mathrm{ano}^{-1}\right.$ de lodo seco); e $2 \mathrm{~L}$ representa a aplicação do dobro da dose recomendada de $\mathrm{N}$ via lodo ( $2 \mathrm{~L} \approx 20 \mathrm{t} \mathrm{ha}^{-1} \mathrm{ano}^{-1}$ de lodo seco). $\mathrm{O}$ delineamento experimental é inteiramente ao acaso e cada tratamento possui quatro repetições, num total de 12 parcelas experimentais com área útil de $100 \mathrm{~m}^{2}$ cada e declive uniforme de $10 \%$. A aplicação de LE nas parcelas foi em área total, com posterior incorporação na camada superficial de $20 \mathrm{~cm}$.

As doses de lodo foram definidas com base na sua análise química (Quadro 1) no primeiro ano de aplicação, na necessidade de $\mathrm{N}$ da cultura (milho) e na taxa de mineralização do $\mathrm{N}$ do resíduo igual a $30 \%$, de acordo com a Norma Técnica P4.230 da Cetesb (Cetesb, 1999). No tratamento AM, foram aplicados anualmente $48 \mathrm{~kg} \mathrm{ha}^{-1} \mathrm{P}_{2} \mathrm{O}_{5}$ e $17 \mathrm{~kg} \mathrm{ha}^{-1}$ de $\mathrm{K}_{2} \mathrm{O}$; enquanto nos tratamentos com lodo somente houve complementação mineral com $\mathrm{K}_{2} \mathrm{O}\left(17 \mathrm{~kg} \mathrm{ha}^{-1} \mathrm{~K}_{2} \mathrm{O}\right)$. Em outubro de 2008 foi feita a última correção da acidez do solo, decorrente do processo de mineralização do lodo, aplicando-se $8 \mathrm{t} \mathrm{ha}^{-1}$ de calcário nas parcelas com lodo.

A área foi cultivada com milho no verão de 2001/ 2002 a 2007/2008, com plantio entre novembro e dezembro de cada ano, após as aplicações do LE. A última aplicação do resíduo foi feita em 21/11/2007. Em 2008/2009 e 2009/2010, após cessarem as aplicações de lodo, o solo continuou a ser cultivado com milho. No verão 2009/2010, o plantio do milho foi feito em outubro e colhido em março de 2010. Em agosto de 2010, foi cultivado milheto; e em março de 2011, foi feito cultivo de girassol.

\section{Amostragem do solo e extração da solução do solo}

Fez-se a amostragem do solo da camada de 0-20 cm, coletando-se oito amostras simples de cada parcela 
Quadro 1. Composição média (base seca) do lodo de esgoto utilizado na área experimental no período de 2001 a 2007

\begin{tabular}{|c|c|}
\hline Componente & Valor médio \pm desvio padrão \\
\hline $\mathrm{pH}$ & $6,6 \pm 0,7$ \\
\hline Umidade (\%) & $68 \pm 3$ \\
\hline Sólido volátel (\%) & $57 \pm 3$ \\
\hline C orgânico $\left(\mathrm{g} \mathrm{kg}^{-1}\right)$ & $279 \pm 43$ \\
\hline N Kjeldahl ( $\left.\mathrm{g} \mathrm{kg}^{-1}\right)$ & $29 \pm 2$ \\
\hline $\mathrm{N}$ amoniacal $\left(\mathrm{mg} \mathrm{kg}^{-1}\right)$ & $371 \pm 133$ \\
\hline $\mathrm{N}$ nitrato-nitrito $\left(\mathrm{mg} \mathrm{kg}^{-1}\right)$ & $48 \pm 52$ \\
\hline $\mathrm{Al}\left(\mathrm{g} \mathrm{kg}^{-1}\right)$ & $19 \pm 2$ \\
\hline $\mathrm{B}\left(\mathrm{mg} \mathrm{kg}^{-1}\right)$ & $24 \pm 25$ \\
\hline $\mathrm{Cd}\left(\mathrm{mg} \mathrm{kg}^{-1}\right)$ & $8,3 \pm 3,6$ \\
\hline $\mathrm{Ca}\left(\mathrm{g} \mathrm{kg}^{-1}\right)$ & $15 \pm 11$ \\
\hline $\mathrm{Pb}\left(\mathrm{mg} \mathrm{kg}^{-1}\right)$ & $170 \pm 61$ \\
\hline $\mathrm{Cu}\left(\mathrm{mg} \mathrm{kg}^{-1}\right)$ & $535 \pm 267$ \\
\hline $\mathrm{Cr}\left(\mathrm{mg} \mathrm{kg}{ }^{-1}\right)$ & $168 \pm 22$ \\
\hline $\mathrm{S}\left(\mathrm{g} \mathrm{kg}^{-1}\right)$ & $20 \pm 6$ \\
\hline $\mathrm{Fe}\left(\mathrm{g} \mathrm{kg}^{-1}\right)$ & $22 \pm 3$ \\
\hline $\mathrm{P}\left(\mathrm{g} \mathrm{kg}^{-1}\right)$ & $8,1 \pm 2,7$ \\
\hline $\operatorname{Mg}\left(\mathrm{g} \mathrm{kg}^{-1}\right)$ & $1,6 \pm 0,2$ \\
\hline $\left.\mathrm{Mn}(\mathrm{mg} \mathrm{kg})^{-1}\right)$ & $606 \pm 116$ \\
\hline Mo $\left(\mathrm{mg} \mathrm{kg}^{-1}\right)$ & $8,3 \pm 2,7$ \\
\hline $\mathrm{Ni}\left(\mathrm{mg} \mathrm{kg}{ }^{-1}\right)$ & $59 \pm 58$ \\
\hline $\mathrm{Zn}\left(\mathrm{mg} \mathrm{kg}{ }^{-1}\right)$ & $1339 \pm 279$ \\
\hline $\mathrm{K}\left(\mathrm{mg} \mathrm{kg}{ }^{-1}\right)$ & $2538 \pm 3944^{(1)}$ \\
\hline $\mathrm{Na}\left(\mathrm{mg} \mathrm{kg}^{-1}\right)$ & $2207 \pm 2613^{(1)}$ \\
\hline $\mathrm{Ba}\left(\mathrm{mg} \mathrm{kg}{ }^{-1}\right)$ & $347 \pm 159$ \\
\hline
\end{tabular}

(1) Em 2004, os valores de K e Na foram 11470 e $8102 \mathrm{mg} \mathrm{kg}^{-1}$, respectivamente.

para obter a amostra composta representativa. As coletas de solo foram realizadas aos $22,26,37$ e 46 meses, após a última aplicação de lodo na área. $\mathrm{Na} 1^{\text {a }}$ e $3^{\mathrm{a}}$ amostragem, o solo encontrava-se em pousio, coberto com restos culturais e plantas espontâneas; na $2^{\text {a }}$, estava cultivado com milho em fase de maturação dos grãos; enquanto, na $4^{\mathrm{a}}$, encontrava-se cultivado com girassol em final de ciclo.

As amostras foram secas ao ar, peneiradas em malha $2 \mathrm{~mm}$ e armazenadas para as análises.

A obtenção da solução do solo foi por meio de extrato aquoso, conforme descrito em Wolt (1994), em que se utilizou relação solo:água de 1:1. Foram realizadas três extrações distintas para determinar as concentrações de $\mathrm{Al}, \mathrm{B}, \mathrm{Ca}, \mathrm{Cd}, \mathrm{Cr}, \mathrm{Cu}, \mathrm{Fe}, \mathrm{Mg}, \mathrm{Mn}$, $\mathrm{Mo}, \mathrm{Ni}, \mathrm{Pb}, \mathrm{Zn}, \mathrm{K}, \mathrm{Na}, \mathrm{P}$ e S por ICP-OES (Varian Vista MPX), com vista axial ou fotometria de chama, de ânions $\left(\mathrm{F}^{-}, \mathrm{Cl}^{-}, \mathrm{NO}_{2}{ }^{-}, \mathrm{NO}_{3}{ }^{-}, \mathrm{PO}_{4}^{-}, \mathrm{SO}_{4}{ }^{2-}\right)$ por cromatografia iônica, de $\mathrm{NH}_{4}{ }^{+}$por espectrometria no UV-VIS, do carbono orgânico dissolvido (COD) por análise elementar e do $\mathrm{pH}$. Antes da determinação do COD, os extratos aquosos foram filtrados em filtro de microfibra de vidro $\mathrm{GF} / \mathrm{F}$ de $47 \mathrm{~mm}$ previamente calcinados a $500{ }^{\circ} \mathrm{C}$ por $6 \mathrm{~h}$. As amostras foram armazenadas em frascos de vidros submetidos às mesmas condições de calcinação dos filtros.

Os cátions foram determinados em ICP-OES e o amônio, em colorímetro UV-Vis de fluxo contínuo (FIA-2500 Flow Injection Analyser), pelo método do pruciato de ferro. Para quantificar os ânions, utilizouse cromatógrafo de íons da marca Metrohm. Determinou-se o COD pelo equipamento Total Organic Carbon Analyzer (TOC-V CPN) da Shimadzu.

As amostras de solo foram ainda submetidas à extração com solução de DTPA em $\mathrm{pH}$ 7,3 para determinar $\mathrm{Cu}$ e $\mathrm{Zn}$, empregando $20 \mathrm{~cm}^{3}$ de solo e $40 \mathrm{~mL}$ da solução extratora, conforme descrito por Abreu et al. (2001).

\section{Análise dos dados}

Os dados, consistindo de uma matriz de 36 linhas (amostras) por 26 colunas (variáveis), correspondentes a cada época de amostragem da solução do solo, foram submetidos inicialmente à análise de correlação de Pearson, com 95 \% de confiança. O objetivo dessa operação foi identificar se há variáveis altamente correlacionadas entre si e, portanto, que apresentam a mesma informação estatística (variância) para o conjunto de dados. Quando isso ocorre uma das variáveis altamente correlacionadas pode ser descartada, reduzindo-se a matriz de dados a ser examinada e permitindo melhor definição dos grupos de amostras obtidos posteriormente na ACP. No conjunto de variáveis resultante, foi aplicada a ACP, centrando os dados na média. Para essa análise, foi utilizado o software XLSTAT.

\section{RESULTADOS E DISCUSSÃO}

\section{Efeito residual do lodo na solução do solo}

Os elevados teores de $\mathrm{Ca}$ e $\mathrm{Mg}$ encontrados na solução do solo dos tratamentos 1L e 2L (Quadro 2) podem ser atribuídos à calagem realizada em 2008 para correção da acidez, bem como ao aporte desses nutrientes via LE (Quadro 1). Comparando-se as concentrações de $\mathrm{Ca}$ e $\mathrm{Mg}$ entre os tratamentos $1 \mathrm{~L}$ e $2 \mathrm{~L}$, evidenciou-se a contribuição do lodo para o incrementos na disponibilidade desses nutrientes. Em média, para as quatro épocas de avaliação, incrementos de 46 e $40 \%$ para $\mathrm{Ca}$ e $\mathrm{Mg}$, respectivamente, ocorreram quando a dose de lodo aplicada dobrou e, nesse caso, excluiu-se o efeito da calagem, uma vez que ambos os tratamentos receberam a mesma dose de corretivo.

Num primeiro momento, valores de $\mathrm{pH}$ mais elevados poderiam ser esperados nos tratamentos com lodo, pois em 2008 esses receberam calcário. No entanto, a mineralização do $\mathrm{N}$ orgânico adicionado ao solo via doses de lodo conduz à formação de $\mathrm{NH}_{4}{ }^{+} \mathrm{e}$, 
em meio aeróbio, $\mathrm{NO}_{3}$ - (Victoria et al., 1992; Andrade et al., 2010), com produção de $2 \mathrm{~mol}$ de $\mathrm{H}^{+}$para cada mol de N nitrificado (Essington, 2003). Do balanço entre o efeito residual da calagem e uma suposta mineralização do $\mathrm{N}$ mais intensa no solo com lodo (Andrade et al., 2013), verificou-se que até aproximadamente 37 meses após a ultima aplicação de lodo e 33 meses após a calagem, os tratamentos $1 \mathrm{~L}$ e $2 \mathrm{~L}$ permaneciam com condição de acidez semelhante ao tratamento AM (Quadro 3). O pH é o fator crítico mais importante ligado à disponibilidade dos nutrientes minerais e elementos potencialmente tóxicos (Adriano, 1986).

Considerando o histórico da área, bem como o tempo decorrido após a última aplicação de lodo, esperava-se que as concentrações das espécies químicas relacionadas ou não com a dinâmica da matéria orgânica se reduzissem com os eventos de amostragem e isso foi, de fato, comprovado (Quadros 2 e 3).

Tanto na transição da $1^{\mathrm{a}}$ para a $2^{\mathrm{a}}$ amostragem (22 para 26 meses após a última aplicação), quanto da $3^{\mathrm{a}}$ para a $4^{\mathrm{a}}$ amostragem (36 para 47 meses da ultima aplicação) ocorreram chuvas intensas e temperaturas relativamente elevadas, características da região, contribuindo tanto para a mineralização da matéria orgânica como para a lixiviação de nutrientes e formas nitrogenadas decorrentes (nitrato, nitrito e amônio). Alem disso, na $2^{\mathrm{a}}$ e na $4^{\mathrm{a}}$ amostragem a área encontrava-se cultivada e as menores concentrações desses elementos na solução do solo podem ser atribuídas especialmente à absorção de nutrientes pelas plantas. $O$ efeito de mineralização sugerido é evidenciado pela diminuição do $\mathrm{pH}$, que foi mais baixo aos 26 e 37 meses, em relação à $1^{\text {a época, }}$

Quadro 2. Concentração média de cátions na solução do solo

\begin{tabular}{|c|c|c|c|c|c|c|c|c|c|c|}
\hline Tempo $^{(1)}$ & $\operatorname{Dose}^{(2)}$ & $\mathrm{Al}^{3+}$ & $\mathrm{Ca}^{2+}$ & $\mathrm{Fe}^{3+}$ & $\mathrm{Mg}^{2+}$ & $\mathrm{Mn}^{2+}$ & $\mathrm{Cu}^{2+}$ & $\mathrm{Zn}^{2+}$ & $\mathrm{Na}^{+}$ & $\mathrm{NH}_{4}{ }^{+}$ \\
\hline mês & & & & & & $\mathrm{mg} \mathrm{L}^{-1}$ & & & & \\
\hline \multirow[t]{3}{*}{22} & $\mathrm{AM}$ & 5,20 & 12,5 & 1,9 & 7,3 & 0,50 & 0,005 & 0,010 & 1,0 & 2,0 \\
\hline & $1 \mathrm{~L}$ & 0,62 & 36,1 & 0,15 & 32,3 & 0,88 & 0,017 & 0,015 & 1,2 & 3,9 \\
\hline & $2 \mathrm{~L}$ & 0,22 & 49,3 & 0,001 & 43,7 & 2,20 & 0,035 & 0,014 & 1,7 & 6,3 \\
\hline \multirow[t]{3}{*}{26} & $\mathrm{AM}$ & 1,30 & 6,1 & 1,3 & 3,2 & 0,14 & 0,040 & 0,009 & 0,8 & 0,7 \\
\hline & $1 \mathrm{~L}$ & 1,50 & 20,6 & 1,1 & 17,0 & 0,30 & 0,012 & 0,024 & 1,1 & 1,2 \\
\hline & $2 \mathrm{~L}$ & 0,29 & 30,0 & 0,04 & 25,8 & 1,20 & 0,025 & 0,180 & 1,2 & 2,6 \\
\hline \multirow[t]{3}{*}{37} & $\mathrm{AM}$ & 1,50 & 10,1 & 1,4 & 5,2 & 0,06 & 0,008 & 0,020 & 1,0 & 0,7 \\
\hline & $1 \mathrm{~L}$ & 0,89 & 28,0 & 0,57 & 23,1 & 0,15 & 0,004 & 0,007 & 1,4 & 1,1 \\
\hline & $2 \mathrm{~L}$ & 0,16 & 46,5 & 0,04 & 34,2 & 1,20 & 0,019 & 0,390 & 2,0 & 1,5 \\
\hline \multirow[t]{3}{*}{46} & $\mathrm{AM}$ & 2,40 & 4,3 & 1,1 & 2,4 & 0,15 & 0,055 & 0,004 & 1,3 & 1,6 \\
\hline & $1 \mathrm{~L}$ & 1,40 & 12,6 & 3,6 & 10,3 & 0,19 & 0,037 & 0,017 & 1,5 & 1,9 \\
\hline & $2 \mathrm{~L}$ & 0,85 & 17,2 & 0,7 & 12,9 & 0,64 & 0,005 & 0,004 & 1,7 & 2,2 \\
\hline
\end{tabular}

(1) Meses decorridos após a ultima aplicação de lodo. ${ }^{(2)}$ AM: adubação mineral; 1L: dose de lodo recomendada; $2 \mathrm{~L}$ : dobro da dose de lodo recomendada com base na cultura do milho.

Quadro 3. Concentração média de ânions e pH na solução do solo

\begin{tabular}{|c|c|c|c|c|c|c|c|c|c|}
\hline Tempo $^{(1)}$ & $\operatorname{Dose}^{(2)}$ & $\mathbf{F}^{-}$ & $\mathrm{Cl}^{-}$ & $\mathrm{NO}_{2}^{-}$ & $\mathrm{NO}_{3}^{-}$ & $\mathrm{PO}_{4}{ }^{3-}$ & $\mathrm{SO}_{4}{ }^{2-}$ & $\mathrm{COD}^{(3)}$ & pH \\
\hline \multicolumn{10}{|c|}{$-\mathrm{mg} \mathrm{L}^{-1}$} \\
\hline \multirow[t]{3}{*}{22} & $\mathrm{AM}$ & 0,09 & 5,1 & 1,1 & 96,9 & 0,20 & 80,7 & 61,3 & 5,2 \\
\hline & $1 \mathrm{~L}$ & 0,01 & 4,5 & 0,5 & 95,4 & 0,10 & 81,5 & 115,0 & 6,0 \\
\hline & $2 \mathrm{~L}$ & 0,01 & 4,4 & 0,7 & 126,1 & 0,70 & 103,0 & 141,0 & 5,4 \\
\hline \multirow[t]{3}{*}{26} & $\mathrm{AM}$ & 0,14 & 4,5 & 0,5 & 28,5 & 0,15 & 11,6 & 32,1 & 4,5 \\
\hline & $1 \mathrm{~L}$ & 0,12 & 4,8 & 0,8 & 50,3 & 0,24 & 72,5 & 50,8 & 5,5 \\
\hline & $2 \mathrm{~L}$ & 0,13 & 4,7 & 0,5 & 75,4 & 0,52 & 146,0 & 63,2 & 4,5 \\
\hline \multirow[t]{3}{*}{37} & $\mathrm{AM}$ & 0,07 & 6,6 & 0,4 & 65,5 & 0,01 & 34,3 & 34,9 & 4,9 \\
\hline & $1 \mathrm{~L}$ & 0,17 & 7,6 & 1,1 & 115,0 & 0,01 & 78,7 & 57,2 & 5,9 \\
\hline & $2 \mathrm{~L}$ & 0,06 & 9,4 & 0,6 & 211,0 & 0,01 & 104,0 & 60,0 & 4,4 \\
\hline \multirow[t]{3}{*}{46} & $\mathrm{AM}$ & 1,6 & 10,0 & 0,01 & 5,6 & 0,01 & 15,2 & 21,9 & 5,2 \\
\hline & $1 \mathrm{~L}$ & 3,2 & 13,1 & 0,07 & 14,4 & 1,60 & 51,3 & 38,6 & 5,7 \\
\hline & $2 \mathrm{~L}$ & 2,3 & 13,1 & 0,01 & 32,2 & 0,04 & 91,4 & 37,7 & 4,9 \\
\hline
\end{tabular}

(1) Meses decorridos após a ultima aplicação de lodo. ${ }^{(2)}$ AM: adubação mineral; 1L: dose de lodo recomendada; $2 \mathrm{~L}$ : dobro da dose de lodo recomendada com base na cultura do milho. ${ }^{(3)}$ COD: carbono orgânico dissolvido. 
mesmo com a calagem efetuada nos tratamentos $1 \mathrm{~L} \mathrm{e}$ 2L em 2008. A exceção a esse padrão foi observada na $3^{\mathrm{a}}$ amostragem, com elevação relativa da concentração de alguns elementos em todos os tratamentos, como o $\mathrm{Ca}$ e $\mathrm{Mg}$, cujo aporte justifica-se pela permanência de restos culturais na área experimental. As concentrações relativas de $\mathrm{SO}_{4}{ }^{2-}, \mathrm{NO}_{3}{ }^{-}$e $\mathrm{COD}$ também aumentaram nesse período, mas não foi possível apontar sua origem, mineralização residual do lodo ou dos mesmos restos culturais.

\section{Disponibilidade de metais pesados}

O lodo aplicado na área ao longo de sete anos apresentou $\mathrm{Cd}, \mathrm{Cr}, \mathrm{Ni}, \mathrm{Pb}, \mathrm{Mo}, \mathrm{Cu}$ e $\mathrm{Zn}$ em concentrações variáveis (Quadro 1). No entanto, as concentrações de $\mathrm{Cd}$, Mo e Ni encontradas no lodo podem ser consideradas baixas, variando de 5 a $12 \mathrm{mg} \mathrm{kg}^{-1}$ para os dois primeiros elementos e de níveis não detectáveis a um máximo de cerca de $120 \mathrm{mg} \mathrm{kg}^{-1}$ para o Ni. Para $\mathrm{Cr}$ e $\mathrm{Pb}$, o lodo apresentou concentrações intermediárias, indo de 100 a cerca de $200 \mathrm{mg} \mathrm{kg}^{-1}$. Já para o $\mathrm{Cu}$, a concentração no lodo variou na faixa de 270 a $800 \mathrm{mg} \mathrm{kg}^{-1} \mathrm{e}$, para Zn, de 1000 a $1500 \mathrm{mg} \mathrm{kg}^{-1}$, o que, considerando as aplicações anuais, representa aporte significativo ao solo.

Os teores de $\mathrm{Cu}$ e de $\mathrm{Zn}$ extraídos pelo método do DTPA em pH 7,3, após 22 meses da última aplicação de lodo, foram: para o $\mathrm{Cu}$, de 5,$1 ; 12,4$; e $20,9 \mathrm{mg} \mathrm{kg}^{-1}$; e, para o Zn, de 2,1; 23,3; e 24,4 mg kg ${ }^{-1}$, considerando, respectivamente, os tratamentos AM, 1L e 2L. Após 46 meses, esses teores foram iguais a 4,8; 10,0; e $12,6 \mathrm{mg} \mathrm{kg}^{-1}$ de $\mathrm{Cu}$; e 0,$9 ; 13,3$; e 12,8 mg kg-1 de Zn, novamente para os tratamentos AM, $1 \mathrm{~L}$ e $2 \mathrm{~L}$. Isso indicou que os estoques desses nutrientes no solo da área, mesmo após chuvas e ciclos das culturas, continuaram em patamares considerados elevados. Apesar disso, $\mathrm{Cu}$ e $\mathrm{Zn}$ foram encontrados em baixas concentrações na solução do solo (Quadro 2), o que implicou que esses elementos não estiveram prontamente disponíveis em qualquer das épocas de amostragem, apesar dos estoques existentes e da acidez do solo.

Esses resultados indicaram que até os 46 meses após a ultima aplicação de lodo não houve risco ambiental imediato, em razão da presença de $\mathrm{Cu}$ e de Zn na forma de espécies químicas solúveis no sistema estudado que pudessem: causar poluição das águas subsuperficiais, atingir teores tóxicos interferindo nos microrganismos presentes no solo ou apresentar efeitos indesejáveis de acumulação nas plantas (Oliveira et al., 2001; Egiarte et al., 2005; Dynia et al, 2006; Nogueira et al., 2010a).

$\mathrm{O}$ acúmulo de metais no solo e a transferência para tecidos vegetais em razão de aplicações sucessivas e, ou, uso de doses excessivas de lodo de esgoto são reportados na literatura (Oliveira \& Mattiazzo, 2001; Marques et al., 2006; Merlino et al., 2010). Os resultados aqui apresentados conduziram a reflexões importantes a respeito de alguns desvios na interpretação dos teores disponíveis de micronutrientes ou metais extraídos por meio de extratores químicos. Isso ocorre porque parte dos metais introduzidos via lodo de esgoto foi redistribuída em frações pouco móveis no solo e, portanto, pouco disponíveis, mas que podem ser mobilizadas com recuperação variável por causa dos extratores empregados nas análises de fertilidade do solo (Nogueira et al., 2010a,b).

\section{Análise de componentes principais}

Na ACP da $1^{\mathrm{a}}$ amostragem, 22 meses após a última aplicação de lodo de esgoto, empregaram-se 14 variáveis após a exclusão daquelas altamente correlacionadas (como Ca e Mg, conforme discutido anteriormente) e daquelas que se encontravam abaixo dos limites de quantificação instrumentais. A variância explicada pelas duas primeiras componentes principais (PCs) foi de 47,6 \%. As variáveis que mais contribuíram na componente principal 1 (CP1) foram $\mathrm{Al}, \mathrm{Fe}, \mathrm{F}^{-}$e $\mathrm{COD}$; e na $\mathrm{CP} 2$ as variáveis que mais contribuíram foram $\mathrm{pH}, \mathrm{Mn}^{2+}, \mathrm{S}_{1} \mathrm{NO}_{3}{ }^{-}, \mathrm{SO}_{4}{ }^{2-} \mathrm{e} \mathrm{PO}_{4}^{3-}$, correspondendo àquelas de maior módulo nos respectivos eixos do gráfico da figura 1-1b.

No correspondente gráfico das amostras (scores) da $1^{a}$ amostragem (Figura 1-1a), pode ser observada a clara separação das amostras de solo correspondentes à adubação mineral, daquelas com aplicação de lodo, na CP1. A separação das amostras (scores) nas CPs pode ser explicada pela contribuição relativa das variáveis originais no cálculo de cada $\mathrm{CP}$ e pelo gráfico de pesos (loadings) (Figura 1-1b). A sobreposição dos dois permite perceber quais variáveis, entre as que mais contribuíram para a CP em questão, levaram a separação de cada grupo de amostras. Nessa amostragem, essa separação deveu-se parcialmente aos maiores teores de $\mathrm{Al}$ e $\mathrm{Fe}$ (somadas representam $40 \%$ da variância retida nessa $\mathrm{CP}$ ), bem como os menores teores de COD (15 \% de variância) em relação aos tratamentos com aplicação de lodo, o que já era esperado. Embora nos tratamentos 1L e 2L haja diferenças quanto à presença dessas variáveis, a CP2 foi a responsável pela separação entre esses tratamentos, especialmente em razão do $\mathrm{pH}$ (variável de maior peso na PC2) da solução do solo (Figura 1-1b). Para 1L, a média de $\mathrm{pH}$ foi de 6,0; e para o tratamento 2L, de 5,4 (Quadro 3). Observou-se que o COD (variável de maior peso na PC1) não foi suficiente para separar esses dois tratamentos, com uma média de 115 e $141 \mathrm{mg} \mathrm{L}^{-1}$ para os tratamentos $1 \mathrm{~L} \mathrm{e} 2 \mathrm{~L}$, respectivamente (Quadro 3). Isso era esperado com base na quantidade de matéria orgânica contida nas doses de lodo aplicadas.

Verificou-se ainda a presença de $\mathrm{F}^{-}$nas amostras $\mathrm{AM}$, contribuindo para a separação desse grupo na $\mathrm{CP} 1$. O F-, junto com $\mathrm{Al}$, $\mathrm{Fe}$ e $\mathrm{COD}$, é uma das variáveis de maior peso na CP1 (Figura 1-1b), deslocando as amostras AM para o lado positivo do gráfico de scores (Figura 1-1a). A adubação fosfatada com superfosfato triplo ou superfosfato simples que 

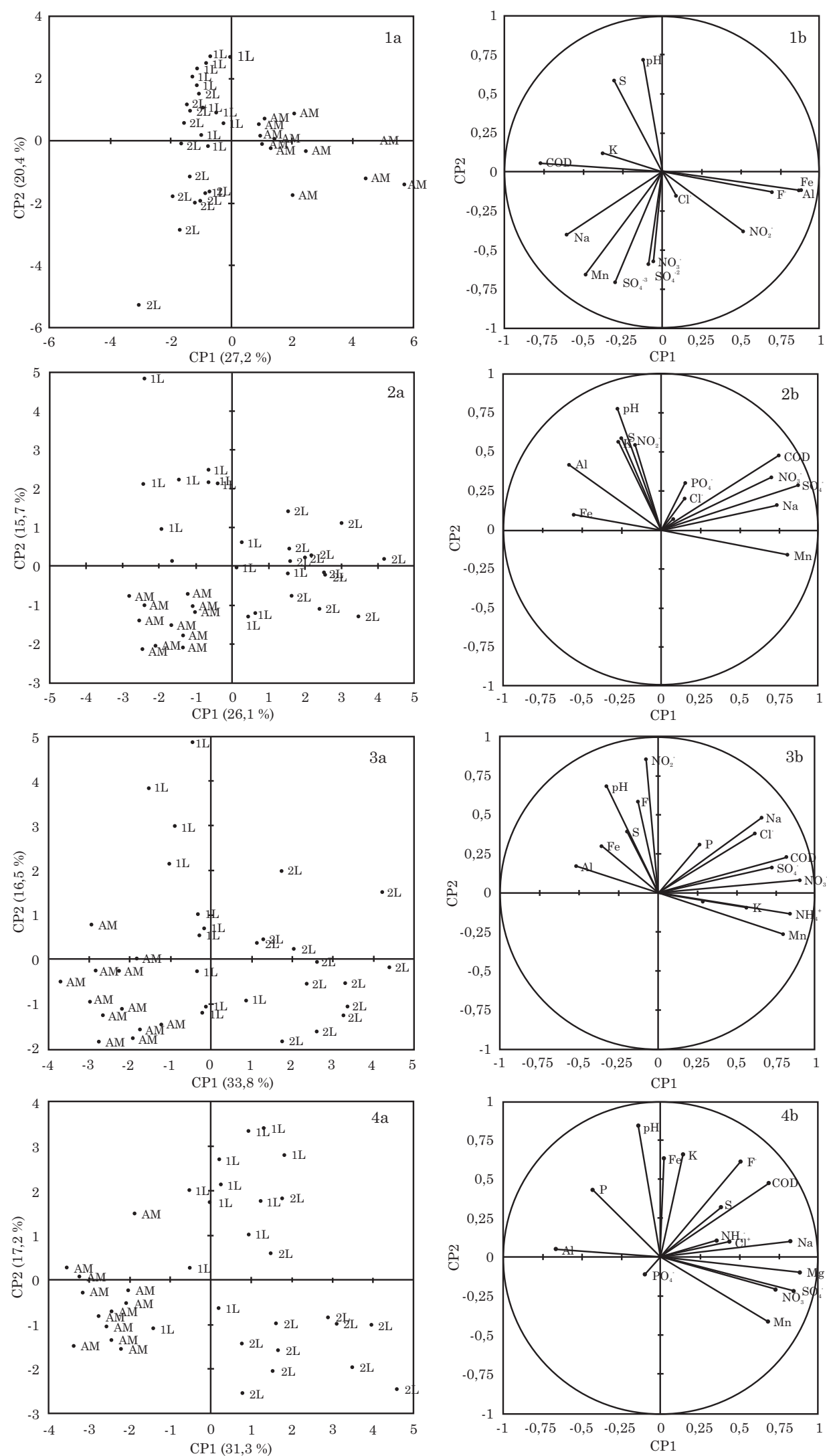

Figura 1. Gráficos de scores (a) e loadings (b) para a análise de componentes principais da solução do solo nas quatro amostragens realizadas. 1) 22 meses; 2) 26 meses; 3) 37 meses; e 4) 46 meses, após a ultima aplicação de lodo na área experimental. AM: adubação mineral; 1L: dose de lodo recomendada; e 2L: dobro da dose de lodo recomendada com base na cultura do milho. 
foi empregada nesse tratamento contém $\mathrm{CaF}_{2}$ em sua composição (Alcarde, 2009).

$\mathrm{Na} 2^{\mathrm{a}}$ amostragem, 26 meses após a última aplicação de LE, a análise por componentes principais evidenciou que 41,8 \% da variância total foram explicadas pelos dois primeiros componentes. As variáveis que mais contribuíram na PC1 $(26,1 \%$ de variância) foram $\mathrm{Mn}^{2+}, \mathrm{Na}^{+}, \mathrm{NO}_{3}{ }^{-}, \mathrm{SO}_{4}^{2-}$ e $\mathrm{COD}$, seguidas por $\mathrm{Al}^{3+} \mathrm{e} \mathrm{Fe}^{3+}$. Na CP2 (15,7\% de variância), as variáveis mais importantes foram $\mathrm{pH}, \mathrm{K}^{+}, \mathrm{Se} \mathrm{NO}_{2}$ (Figura 1-2b). Nessa amostragem, ocorreu maior distinção dos três tratamentos na $\mathrm{PC} 1$, em comparação com a $1^{\text {a }}$ amostragem, o que se ampliou até a $4^{\mathrm{a}}$ amostragem (46 meses após a última aplicação de lodo) (Figura 1-2a). Isso significou menor complexidade dos dados com o tempo de amostragem, uma vez que um menor número de variáveis foi necessário para obter essa separação, o que foi consistente com os processos de perdas e redução do efeito residual do lodo e da calagem, conforme discutido anteriormente. Dessa forma, observou-se que algumas variáveis responsáveis pela separação entre tratamentos na $1^{\mathrm{a}}$ época de amostragem perdem significância (menor variância); e para que a separação dos tratamentos seja possível, outras variáveis passam a ser consideradas na análise de componentes principais. Esse é o caso, por exemplo, do $\mathrm{Na}$ (13\% da variância na PC1), cuja concentração pouco variou na solução do solo, ao longo do tempo, dentro de cada tratamento (Quadro 2). Esse elemento não foi relacionado com a dinâmica da matéria orgânica no solo, também não foi um nutriente de plantas e foi encontrado no lodo em baixa concentração, semelhante ao K. A concentração de $\mathrm{Na}^{+}$associada aos tratamentos com lodo não se deve exclusivamente ao $\mathrm{Na}^{+}$presente no lodo, mas ao incremento na CTC do solo (Bueno et al., 2011), reduzindo as perdas de cátions em geral por lixiviação, principalmente de cátions monovalentes $\left(\mathrm{Na}^{+}\right)$, cuja força de retenção é menor do que dos cátions divalentes e trivalentes (série liotrópica).

Destacou-se a presença de $\mathrm{NO}_{2}{ }^{-}$nas amostras dos tratamentos $1 \mathrm{~L}$ e $2 \mathrm{~L}$ na $2^{\mathrm{a}}, 3^{\mathrm{a}}$ e $4^{\mathrm{a}}$ amostragem. A variação das concentrações de $\mathrm{NO}_{2}{ }^{-}$seguiu as alterações observadas para o $\mathrm{NO}_{3}^{-}$, ou seja, seguiram a dinâmica da nitrificação da matéria orgânica no solo ao longo do período em estudo.

$\mathrm{Na} 3^{\mathrm{a}}$ época de amostragem, a análise por componentes principais evidenciou que 50,3\% da variância total foram explicadas pelos dois primeiros componentes. As variáveis que apresentaram maior influência na CP1 foram $\mathrm{NH}_{4}{ }^{+}, \mathrm{Mn}^{2+}, \mathrm{COD}, \mathrm{NO}_{3}{ }^{-} \mathrm{e}$ $\mathrm{SO}_{4}^{2-} ; \mathrm{e}$, na $\mathrm{CP} 2$, as variáveis mais importantes foram $\mathrm{NO}_{2}^{-}$e $\mathrm{pH}$ (Figura 1-3a).

$\mathrm{Na} 4^{\mathrm{a}}$ época de amostragem, 46 meses após a última aplicação de LE, a análise por componentes principais evidenciou que 48,5 \% da variância total verificada foram explicadas pelos dois primeiros componentes. As variáveis com maior influência na $\mathrm{CP} 1$ foram $\mathrm{Mg}^{2+}, \mathrm{Mn}^{2+}, \mathrm{Na}^{+}, \mathrm{NO}_{3}{ }^{-}, \mathrm{SO}_{4}{ }^{2-}$ e COD. $\mathrm{Na}$
$\mathrm{CP} 2$, as variáveis mais importantes foram $\mathrm{pH}, \mathrm{K}^{+}$, $\mathrm{Fe}^{3+}$ e F- (Figura 1-4a).

Embora se tenham observado diferenças entre as variáveis com maior contribuição para os dois primeiros componentes principais, algumas apareceram de forma consistente nas quatro épocas de avaliação, sendo essas diretamente relacionadas com a decomposição da matéria orgânica, como $\mathrm{NH}_{4}{ }^{+}$, $\mathrm{NO}_{2}-\mathrm{NO}_{3}^{-}, \mathrm{SO}_{4}{ }^{2-}, \mathrm{PO}_{4}^{3-}, \mathrm{Mn}^{2+}, \mathrm{pH}$ e COD.

A importância do efeito residual da degradação da matéria orgânica encontrada neste estudo também tem sido apontada em outros trabalhos realizados nessa mesma área experimental e, em conjunto com este, indicam a necessidade de contemplar o manejo de áreas com aplicações sucessivas de lodo de esgoto em áreas agrícolas na legislação existente hoje. No trabalho de Andrade et al. (2013), avaliaram-se em laboratório a degradação do $\mathrm{C}$ e mineralização do $\mathrm{N}$ no solo da área experimental (um ano após a última aplicação do lodo) em razão de novas doses de LE aplicadas. Concluiu-se que ainda havia um remanescente do lodo das aplicações anteriores e que a velocidade de mineralização do $\mathrm{N}$ recentemente aplicado foi alterada em relação aos históricos de uso do lodo, com maior potencial de fornecimento de $\mathrm{N}$ às plantas nos solos dos tratamentos com uso anterior de lodo, aumentando, inclusive, o risco de lixiviação de $\mathrm{NO}_{3}$. Pitombo (2011) determinou os estoques de $\mathrm{C}$ e $\mathrm{N}$ e o efeito residual das aplicações de lodo ou fertilizante nitrogenado mineral nas emissões de $\mathrm{CO}_{2}$, $\mathrm{N}_{2} \mathrm{O}$ e $\mathrm{CH}_{4}$, a partir de solo. Esse autor reportou que as aplicações sucessivas de lodo proporcionaram incrementos de $\mathrm{C}$ e $\mathrm{N}$ no solo, e apesar das emissões dos gases do efeito estufa terem aumentado, com o lodo houve um balanço mais favorável de $\mathrm{C}$ no sistema.

\section{CONCLUSÕES}

1. As determinações analíticas realizadas em amostras da solução do solo e o uso da análise por componentes principais permitem distinguir áreas com adubação mineral e áreas fertilizadas com lodo de esgoto.

2. Alguns atributos da solução do solo podem ser utilizados para monitoramento do efeito residual do lodo de esgoto no solo agrícola, sendo aqueles relacionados à matéria orgânica os mais indicados para essa finalidade: $\mathrm{pH}, \mathrm{Mn}^{2+}$, $\mathrm{COD}, \mathrm{SO}_{4}{ }^{2-}, \mathrm{NO}_{3}-\mathrm{e} \mathrm{NH}_{4}{ }^{+}$.

\section{LITERATURA CITADA}

ABREU, C.A.; ABREU, M.F. \& ANDRADE, J.C. Determinação de cobre, ferro, manganês, zinco, cádmio, cromo e chumbo em solos usando a solução de DTPA em pH 7,3 In: RAIJ, B.van; ANDRADE, J.C.; CANTARELLA, H. \& QUAGGIO, J.A., eds. Análise química para avaliação da fertilidade de solos tropicais. Campinas, Instituto Agronômico de campinas, 2001. p.240-250. 
ADRIANO, D.C. Trace elements in the terrestrial environment. New York, Springer-Verlag, 1986. 533 p.

ALCARDE, J.C. Manual de análise de fertilizantes. Piracicaba, FEALQ, 2009. 259p.

ANDRADE, C.A.; BOEIRA, R.C. \& PIRES, A.M.M. Nitrogênio presente em LE e a Resolução $\mathrm{n}^{\circ} 375$ do Conama. In: COSCIONE, A.R.; NOGUEIRA, T.A.R. \& PIRES, A.M.M., orgs. Uso Agrícola de LE: Avaliação após Resolução ${ }^{\circ}$ 375 do Conama. Botucatu, 2010. p.157-169.

ANDRADE, C.A.; SILVA, L.F.M.; PIRES, A.M.M. \& COSCIOnE, A.R. Mineralização do carbono e do nitrogênio no solo apos sucessivas aplicações de lodo de esgoto. Pesq. Agropec. Bras., 48:536- 544, 2013.

BETTIOL, W. \& CAMARGO, O.A. A disposição de LE em solo agrícola. In: BETTIOL, W. \& CAMARGO, O., eds. LE: Impactos ambientais na agricultura. Jaguariúna, Embrapa Meio Ambiente, 2006. p.25-35.

BUENO, J.R.P.; BERTON, R.S.; SILVEIRA, A.P.D.; CHIBA, M.K.; ANDRADE, C.A. \& DE MARIA, I.C. Chemical and microbiological attributes of an Oxisol treated with successive applications of sewage sludge. R. Bras. Ci. Solo, $35: 1461-1470,2011$.

CAMARGO, O.A. \& BETTIOL, W. De resíduo a fertilizante: Uma análise prospectiva do meio ambiente. In: COSCIONE, A.R.; NOGUEIRA, T.A.R. \& PIRES, A.M.M., orgs. Uso agrícola de lodo de esgoto: Avaliação após a resolução no. 375 do Conama. Botucatu, FEPAF, 2010. p.13-30.

COMPANHIA DE TECNOLOGIA DE SANEAMENTO AMBIENTAL - CETESB. Aplicação de biossólidos de sistemas de tratamento biológico em áreas agrícolas Critérios para projeto e operação: Manual técnico. São Paulo, 1999. 33p.

CONSELHO NACIONAL DE MEIO AMBIENTE - CONAMA. Resolução $\mathrm{n}^{\circ}$ 375/2006. Brasília, 2006. Disponível em: <ht tp://www.mma.gov.br/port/conama/ legiabre.cfm?codlegi=506>. Acesso em: 19 maio 2014.

DE MARIA, I.C.; KOCSSI, M.A. \& DECHEN, S.C.F. Agregação do solo em área que recebeu LE. Bragantia, 66:291-298, 2007.

DYNIA, J.F.; SOUZA, M.D. \& BOEIRA, R.C. Lixiviação de nitrato em Latossolo cultivado com milho após aplicações sucessivas de lodo de esgoto. Pesq. Agropec. Bras., 41:855$862,2006$.

EGIARTE, G.; ARBESTAIN, M.C.; ALONSO, A.; RUIZROMERA, E. \& PINTO, M. Effect of repeated applications of sewage sludge on the fate of $\mathrm{N}$ in soils under Monterey pine stands. For. Ecol. Manage., 216:257-269, 2005.

EMPRESA BRASILEIRA DE PESQUISA AGROPECUÁRIA EMBRAPA. Sistema brasileiro de classificação de solos. Brasília, Embrapa-SPI; Rio de Janeiro, Embrapa Solos, 2006. 306p.
ESSINGTON, M.E. Soil and water chemistry: An integrative approach. Boca Raton, CRC Press, 2003. 534p.

HADLICH, G.M. \& UCHA, J.M. Distribution of cadmium in a cultivated soil in Britanny, France. Sci. Agric., 67:731736, 2010.

LYRA, W.S.; SILVA, E.C.; ARAÚJO, M.C.U.; FRAGOSO, W.D. \& VERAS, G. Classificação periódica: um exemplo didático para ensinar análise de componentes principais. Química Nova, 33:1594-1597, 2010.

MARQUES, M.O.; NOGUEIRA, T.A.R.; FONSECA, I.M. \& MARQUES, T.A. Metais pesados em solo tratado com LE e cultivado com cana-de-açúcar. Colloq. Agrar., 2:46-56, 2006.

MERLINO, L.C.S.; MELO, W.J.; MACEDO, F.G.; GUEDES, A.C.T.P.; RIBEIRO, M.H.; MELO, V.P. \& MELO, G.M.P. Bário, cádmio, cromo e chumbo em plantas de milho e em Latossolo após onze aplicações anuais de LE. R. Bras. Ci. Solo, 34:2031-2039, 2010.

NOGUEIRA, T.A.R.; MELO, W.J.; FONSECA, I.M.; MARQUES, M.O. \& HE, Z. Barium uptake by maize plants as affected by sewage sludge in a long-term field study. J. Hazard. Mater., 181:1148-1157, 2010a.

NOGUEIRA, T.A.R.; MELO, W.J.; FONSECA, I.M.; MARCUSSI, S.A. \& MELO, G.M.P. Fractionation of Zn, $\mathrm{Cd}$ and $\mathrm{Pb}$ in a tropical soil after nine-year sewage sludge applications. Pedosphere, 20:545-556, 2010b.

OLIVEIRA, F.C.; MATTIAZZO, M.E.; MARCIANO, C.R. \& MORAES, S.O. Lixiviação de nitrato em um Latossolo Amarelo distrófico tratado com lodo de esgoto e cultivado com cana-de-açúcar. Sci. Agric., 58:171-180, 2001.

OLIVEIRA, F.C. \& MATTIAZZO, M.E. Metais pesados em Latossolo tratado com lodo de esgoto em plantas de cana de açúcar. Sci. Agric., 58:581-593, 2001.

PITOMBO, L.M. Estoques de carbono e nitrogênio, qualidade da matéria orgânica e fluxos de $\mathrm{CO}_{2}, \mathrm{CH}_{4}$ e $\mathrm{N}_{2} \mathrm{O}$ em solo com diferentes históricos de aplicação de LE. Campinas, Instituto Agronômico, 2011. 61p. (Dissertação de Mestrado)

SIMONETE, M.A.; KIEHL, J.C.; ANDRADE, C.A. \& TEIXEIRA, C.F.A. Efeito do LE em um Argissolo e no crescimento e nutrição de milho. Pesq. Agropec. Bras., 10:1187-1195. 2003.

VALLADARES, G.S.; SANTOS, G.C.G.; ABREU, C.A.; CAMARGO, O.A. \& FERRERO, J.P. Zinco total e disponível em amostras de perfis de solos do estado de São Paulo. Bragantia, 68:1105-1114, 2009.

VICTORIA, R.L.; PICCOLO, M.C. \& VARGAS, AA.T. O ciclo do nitrogênio. In: CARDOSO, E.J.B.N.; TSAI, S.M. \& NEVES, M.C.P., eds. Microbiologia do solo. Campinas, Sociedade Brasileira de Ciência do Solo, 1992. p.105-119.

WOLT, J.D. Soil solution chemistry: Applications to environmental science and agriculture. New York, John Wiley, 1994. 345p. 